\title{
Íleo paralítico em três bovinos: relato de caso*
}

\section{Paralytic ileus in three cattle: case report}

\author{
Lucas da Costa Dutra, ${ }^{* *}$ Luiz Teles Coutinho, ${ }^{* *}$ Carla Lopes de Mendonça, ${ }^{* *}$ Rodolfo José Cavalcanti Souto, ${ }^{* *}$ \\ Jobson Filipe de Paula Cajueiro, ${ }^{* *}$ Nivaldo de Azevêdo Costa, ${ }^{* *}$ José Augusto Bastos Afonso**
}

\begin{abstract}
Resumo
O íleo paralítico é uma obstrução do tipo funcional, na qual o lúmen intestinal está patente, comprometendo a passagem da ingesta no intestino. Este relato objetiva descrever três casos em vacas com aptidão leiteira, atendidos na rotina hospitalar e diagnosticados com íleo paralítico. Em seus históricos, os proprietários queixavam-se que os animais apresentavam redução do apetite, timpania ruminal, diminuição da produção de leite e eliminação das fezes. Ao exame físico, alguns sinais clínicos apresentaram destaque, como apatia, desidratação, redução da motilidade ruminal e intestinal, fezes em pequena quantidade e com muco, distensão do abdômen e ao balotamento constatou-se a presença de líquido. Na análise do fluido ruminal todos os animais apresentaram o teor de cloreto elevado (>30 mEq/L), caracterizando, dessa forma, um processo obstrutivo. Diante dos achados, suspeitou-se inicialmente de um quadro de obstrução intestinal. Nos casos, a conduta adotada foi realizar uma laparotomia exploratória através do flanco direito, porém constatou-se, que não existia qualquer segmento com obstrução de natureza mecânica, que justificasse as alterações físicas e laboratoriais encontradas. Diante destes resultados, configurou-se um quadro clínico indicativo de íleo paralítico. As vacas foram submetidas a um protocolo terapêutico pós-cirúrgico composto por antibioticoterapia, anti-inflamatório, cálcio, procinético e tratamento de suporte. Os animais manifestaram uma resposta favorável a conduta terapêutica, com restabelecimento da função gastrointestinal e dos demais parâmetros fisiológicos, recebendo alta após uma evolução clínica variando entre dez a doze dias.
\end{abstract}

Palavras-Chaves: Afecções intestinais, Distúrbio digestivo, Obstrução funcional, ruminante.

\begin{abstract}
Paralytic ileus is an obstruction of the functional type, in which the intestinal lumen is patent, compromising the passage of the intake in the intestine. The objective of this study was to describe three cases in dairy cows treated in the hospital routine diagnosed with paralytic ileus. In their histories, the owners complained that the animals presented reduced appetite, ruminal tympany, decreased milk yield and elimination of faeces. At the physical examination, some clinical signs were prominent in both, such as apathy, dehydration, reduction of ruminal and intestinal motility, faeces were present in small quantity and with mucus present, abdominal enlargement and the succession produced sloshing sounds. In the analysis of the ruminal fluid, the chloride content in both was high (>30 mEq/L), characterizing an obstructive process. In the face of the findings, a diagnosis of intestinal obstruction was initially suspected. In animals, the adopted approach was to perform an exploratory laparotomy through the right flank, but it was verified that there was no segment with mechanical obstruction that justified the physical and laboratorial alterations found. In view of these results, a clinical diagnosis indicative of paralytic ileus was established. The three animals were submitted to a postsurgical therapeutic protocol consisting of antibiotic therapy, anti-inflammatory, calcium, pro-kinetic and supportive treatment. The animals showed a favourable response to therapeutic treatment, with restoration of gastrointestinal function and other physiological parameters, and was discharged after a clinical evolution ranging from ten to twelve days.
\end{abstract}

Keywords: Digestive disorder, functional obstruction, intestinal disorders, ruminant.

\section{Introdução}

As obstruções intestinais são condições nas quais afetam o trânsito da ingesta através do trato intestinal, reduzindo ou cessando totalmente o seu fluxo. Frequentemente, acomete os bovinos de todas as idades, sendo capaz de levar à morte caso não sejam instituídos o diagnóstico e o tratamento correto (Constable et al., 2017). As obstruções podem ser classificadas em físicas e funcionais. As físicas, nas quais são mais frequentes em bovinos, se caracterizam por uma oclusão física do lúmen intestinal, podendo esta oclusão ser de origem intraluminal ou extraluminal, sendo as principais: fitobezoários, intussuscepção, vólvulo, torção de mesentério, linfoma e linfossarcoma (Hussain et al., 2015; Afonso, 2017; Constable at al., 2017). As obstruções funcionais têm uma menor ocorrência, se caracterizando pelo lúmen intestinal estar patente, porém não ocorre o trânsito da ingesta devido a um problema funcional presente no intestino Dentre estas, destaca-se a ocorrência do íleo paralítico, na qual

*Recebido em 11 de julho de 2018 e aceito em 30 de julho de 2020.

**Unidade Acadêmica de Garanhuns, Universidade Federal Rural de Pernambuco - UFRPE, Garanhuns/PE. 
vêm acometendo habitualmente vacas leiteiras, entretanto, a sua etiopatogenia é pouco descrita na literatura (Pearson e Pinsent, 1977; Pradhan et al., 2016). A sintomatologia apresentada pelos animais acometidos com íleo paralítico são comuns em animais que apresentam algum tipo de transtorno gastrointestinal obstrutivo, não apresentando sinais patognomônicos, consistindo na maioria das vezes em apatia, inapetência/anorexia, redução da motilidade ruminal, hipomotilidade ou atonia intestinal e diminuição ou ausência das fezes, na qual pode evidenciar a presença de muco (Francoz e Guard, 2014).

O objetivo deste relato é descrever os achados clínicos em três bovinos acometidos por íleo paralítico, visto que relatos sobre esse tipo de obstrução são escassos. Em função disso foram abordados os aspectos clínicos-epidemiológicos e a conduta terapêutica empregada em cada caso.

\section{Relato dos casos}

Relatam-se três casos de bovinos atendidos na Clínica de Bovinos de Garanhuns - Campus da Universidade Federal Rural de Pernambuco (CBG/UFRPE), durante o ano de 2017 e 2019 , apresentando transtorno digestivo como queixa principal. O primeiro caso (A1) ocorreu no mês de abril, no ano de 2017, próximo ao início do período chuvoso, acometendo uma fêmea de cinco anos, girolanda, criada em sistema semi-intensivo e parida há 90 dias. Sua alimentação era composta por bagaço de cana (Saccharum officinarum), palma forrageira (Opuntia $s s p)(20 \mathrm{~kg} / \mathrm{dia})$ e concentrado produzido na propriedade a base de milho e soja (6kg/dia). Durante a anamnese, o proprietário relatou que o animal desde o dia anterior estava apático, sem alimentar-se, sem ruminar e apresentando timpania e que era caso único dentro do rebanho. O proprietário ainda relatou que medicou o animal na propriedade com bicarbonato de sódio (VO) e complexo de aminoácidos (mercepton ${ }^{\circledR}$, bravet, Rio de Janeiro, Brasil) (IV). O segundo caso (A2) ocorreu no mês de dezembro, no ano de 2017, período seco na região, acometendo um bovino adulto, fêmea, girolanda, criada em sistema semi-intensivo e parida há 60 dias. Sua alimentação era composta por capim elefante (Pennisetum purpureum), mandioca (Manihot esculenta) (6kg/dia) e concentrado produzido na propriedade a base de milho e soja ( $4 \mathrm{~kg} / \mathrm{dia})$. O proprietário relatou que o animal a cinco dias vinha apresentando quadros de timpania, sendo relatado ainda redução do volume das fezes e aspecto pegajoso das mesmas, citado como caso único dentro do rebanho. Segundo o proprietário, o animal foi medicado com $50 \mathrm{ml}$ de um complexo de aminoácidos (mercepton, bravet, Rio de janeiro, Brasil) (VO) mais 5 litros de solução fisiológica (IV) e $50 \mathrm{ml}$ de acetil tributil acetato (Blo-trol ${ }^{\circledR 1}$, zoetis, São Paulo, Brasil) (VO).

O terceiro caso (A3) ocorreu no final do mês de outubro, no ano de 2019, início do período seco na região, acometendo uma fêmea bovina, da raça holandesa, com três anos de idade (1a lactação), criada em sistema intensivo e parida há 90 dias. Sua alimentação era composta por silagem de sorgo (16 kg/dia), caroço de algodão (2kg/dia), resíduo de cervejariabagaço (12 kg/dia), palma forrageira (Opuntia sp.) (12 kg/dia) e concentrado produzido na propriedade $(8 \mathrm{~kg} / \mathrm{dia})$. O proprietário relatou que no mesmo dia de enviou a CBG/UFRPE, notou o animal com cólicas e escoiceando o abdômen. Medicou com analgésico (Neglumine, Hertape, Minas gerais, Brasil) (IV) e observou que houve melhora, entretanto, durante a tarde os episódios de cólicas se repetiram, mesmo realizando a mesma medicação. Era caso único no lote onde se encontrava. Os três animais descritos neste estudo não apresentaram o quadro anteriormente ao relatado pelo proprietário, bem como foram os primeiros casos em suas respectivas localidades.

Foi realizado exame clínico e análise do fluido ruminal dos animais de acordo com o modelo proposto por Dirksen et al. (1993). Amostras de sangue foram obtidas através da venopunção da veia jugular com tubo a vácuo contendo EDTA (Tubo EDTA K3 vacuette ${ }^{\circledR} 4 \mathrm{ml}$, Turquia) para realização de hemograma de acordo com o método proposto por Jain (1993).

Os achados clínicos dos animais estão descritos na tabela 1. Em especial, apresentavam comportamento de calmo a apático, desidratação moderada, taquicardia, inapetência e anorexia, timpania de branda a moderada, hipomotilidade intestinal e redução na eliminação das fezes. O exame retal evidenciou alças intestinais distendidas e, fezes escassas, fétidas e com muco. Os bovinos A1 e A3 apresentaram ressonância metálica na auscultação com percussão da região dorsal do flanco direito, presença de som de chapinhar em líquido ao balotamento, com abdômen distendido e tensão da parede abdominal pouco aumentada. Ainda, constatou-se um quadro de pneumonia aspirativa no $\mathrm{A} 1$, possivelmente devido à administração oral de bicarbonato de sódio, feita pelo proprietário, sendo encontrado como sinais principais durante o exame físico um animal com respiração taquipneica e polipnéica, apresentando dispneia mista e durante a auscultação pulmonar evidenciou áreas de hipofonese e abafamento em ambos os campos pulmonares, bem como presença de crepitação em regiões crânio-ventrais de ambos campos pulmonares.

No hemograma dos animais A1 e A2 constatou-se leucocitose por neutrofilia com desvio a esquerda regenerativo, além de hiperproteinemia e hiperfibrinogenemia, enquanto no A3 havia uma leucocitose por linfocitose e desvio regenerativo. O exame de urina foi negativo para a presença de corpo cetônico (Tabela 2). Na análise do fluido ruminal, havia comprometimento da microbiota ruminal. Entretanto, destaca-se o resultado do teor de cloretos que foi de $69,93 \mathrm{mEq} / \mathrm{L}, 105,0 \mathrm{mEq} / \mathrm{L}$ e $35,1 \mathrm{mEq} / \mathrm{L}$, no A1, A2 e A3, respectivamente (Tabela 3). 
Tabela 1: Achados clínicos nos três bovinos fêmeas diagnosticados com íleo paralítico

\begin{tabular}{|c|c|c|c|}
\hline Achados Clínicos & A1 & A2 & A3 \\
\hline Atitude & Estação / Posição ortopédica & Estação & Estação \\
\hline Comportamento & Apático & Apático & Calmo \\
\hline Escore nutricional & III & ॥ & III $1 / 2$ \\
\hline Temperatura retal & $38,3^{\circ} \mathrm{C}$ & $37,2^{\circ} \mathrm{C}(\downarrow)$ & $39,4^{\circ} \mathrm{C}(\uparrow)$ \\
\hline Grau de desidratação & Excicose grau III & Excicoce grau II & Excicoce grau I \\
\hline Mucosas & Levemente congestas & Rosadas & Rosadas \\
\hline Vasos episclerais & Levemente Injetados & Levemente Injetados & Injetados \\
\hline Frequência cardíaca & $100 \mathrm{bpm}(\uparrow)$ & 100 bpm $(\uparrow)$ & 104 bpm $(\uparrow)$ \\
\hline $\begin{array}{l}\text { Frequência } \\
\text { respiratória }\end{array}$ & $60 \operatorname{mrpm}(\uparrow)$ & 20 mrpm & $56 \mathrm{mrpm}(\uparrow)$ \\
\hline $\begin{array}{l}\text { Intensidade da } \\
\text { respiração }\end{array}$ & Polipnéica & Oligopnéica & Polipnéica \\
\hline Dispneia & Mista & Ausente & Ausente \\
\hline Ausculta pulmonar & $\begin{array}{l}\text { Presença de crepitação nas regiões } \\
\text { crânio-ventrais de ambos os antímeros } \\
\text { com presença de hipofonese }\end{array}$ & Sem alteração & Sem alteração \\
\hline Apetite & Ausente & Reduzido & Ausente \\
\hline Peristaltismo ruminal & Ausente & Hipomotílico & Hipomotílico \\
\hline Timpania ruminal & Moderada & Moderada & Ausente \\
\hline Formato abdominal & distensão abdominal & distensão abdominal & distensão abdominal \\
\hline Motilidade intestinal & Hipomotílico & Hipomotílico & Hipomotílico \\
\hline $\begin{array}{l}\text { Ausculta com } \\
\text { percussão na Fossa } \\
\text { paralombar direita }\end{array}$ & $\begin{array}{l}\text { Presença de ressonância metálica, com } \\
\text { presença de chapinhar em líquido ao } \\
\text { balotamento. }\end{array}$ & Sem alteração & $\begin{array}{l}\text { Presença de ressonância metálica, } \\
\text { com presença de chapinhar em } \\
\text { líquido ao balotamento. }\end{array}$ \\
\hline Fezes & Diminuídas e com muco & Diminuídas e mal digeridas & Diminuídas e mal digeridas \\
\hline
\end{tabular}

Tabela 2: Valores hematológicos, proteína plasmática total e fibrinogênio plasmático obtidos dos três bovinos fêmeas com diagnóstico de íleo paralítico

\begin{tabular}{lllll}
\hline & Animal 1 & Animal 2 & Animal 3 & Valores de referência* \\
\hline Hemácias $\left(\times 10^{6} / \mu \mathrm{L}\right)$ & 8,15 & 6,71 & 7,3 & $(5,0-10,0)$ \\
Hematócrito $(\%)$ & 29 & 37 & 36 & $(24-46)$ \\
Hemoglobina $(\mathrm{g} / \mathrm{dL})$ & 10,6 & 12,05 & 12,59 & $(8,0-15,0)$ \\
VCM $(\mathrm{fL})$ & 35,58 & 55,1 & 49,31 & $(40,0-60,0)$ \\
CHCM $(\%)$ & 36,5 & 32,45 & 34,97 & $(30,0-36,0)$ \\
Proteína plasmática total $(\mathrm{g} / \mathrm{dL})$ & 8,9 & 9,1 & 7,4 & $(7,0-8,5)$ \\
Fibrinogênio plasmático $(\mathrm{mg} / \mathrm{dL})$ & 800 & 1.100 & 500 & $(300-700)$ \\
Leucócitos totais $(\mu \mathrm{L})$ & 14.700 & 16.350 & 14.900 & $(4.000-12.000)$ \\
Neutrófilos segmentados $(\mu \mathrm{L})$ & 9.849 & 10.627 & 1.192 & $(600-4.000)$ \\
Bastonetes $(\mu \mathrm{L})$ & 1.911 & 164 & 596 & $(0-120)$ \\
Linfócitos $(\mu \mathrm{L})$ & 2.499 & 5.232 & 13.112 & $(2.500-7.500)$ \\
Monócitos $(\mu \mathrm{L})$ & 411 & 163 & 0 & $(25-840)$ \\
Eosinófilos $(\mu \mathrm{L})$ & 0 & 164 & 0 & $(0-2.400)$ \\
\hline
\end{tabular}

*Jain (1993). 
Tabela 3: Análise de fluido ruminal dos três bovinos fêmeas diagnosticados com íleo paralítico

\begin{tabular}{lllll}
\hline & Animal 1 & Animal 2 & Animal 3 & Valores de referência* \\
\hline Cor & Castanho & Castanho claro & Castanho escura & Verde oliva a acastanhado \\
Odor & Alterado & Alterado & Alterado & Aromático \\
Consistência & Levemente Viscosa & Aquosa & Aquosa & Viscosa \\
PH & 6,0 & 6,0 & 6,0 & $6-7$ (pastagens), 5,5-6,5(concentrado) \\
\% de infusórios vivos & $60 \%$ & $0 \%$ & $40 \%$ & $90-100 \%$ \\
Distribuição dos infusórios & $\mathrm{P}^{*}, \mathrm{M}, \mathrm{G}$ & --- & $\mathrm{P}, \mathrm{M}^{*}, \mathrm{G}$ & $\mathrm{P}, \mathrm{M}, \mathrm{G}$ \\
Densidade & ++- & --- & +++ \\
Motilidade & +++ & --- & ++ & +++ \\
PRAM & 4 minutos & Não ocorreu & $2: 30$ minutos & $3-6$ minutos \\
TAS & $>10$ minutos & 8 & Não realizado & $4-8$ minutos \\
FLOT & $>10$ minutos & Não ocorreu & Não ocorreu & $4-8$ minutos \\
\hline Teor de cloreto $(\mathrm{mEq} / \mathrm{L})$ & 69,93 & 105,0 & 35,18 & $<30$ mEq/L \\
\hline
\end{tabular}

*Dirksen et al. (1993)

$P^{*}$ - Predomínio dos infusórios pequenos.

$\mathrm{M}^{*}$ - Predomínio dos infusórios médios.

Diante dos achados clínicos e laboratoriais suspeitou-se de um quadro de obstrução intestinal nos animais, principalmente devido a evidência do teor de cloreto elevado (>30 mEq/L) na análise do fluido ruminal. Inicialmente suspeitou de uma obstrução do tipo física, comumente encontrada na região, porém não descartou obstruções do tipo funcional como íleo paralitico ou indigestão vagal. Os animais foram submetidos a laparotomia exploratória através do flanco direito de acordo com o método proposto por Fubini e Ducharme (2017). Na celiotomia, ao explorar as alças intestinais e demais órgãos, constatou-se a ausência de qualquer elemento intraluminal ou extraluminal, que fosse responsável pelo transtorno digestivo e obstrutivo de natureza física. Nenhuma evidência de motilidade intestinal - delgado e grosso - foi observada durante o procedimento cirúrgico. Com isso, os achados do exame físico aliados aos encontrados durante a celiotomia levantou a hipótese da ocorrência de um quadro de obstrução intestinal do tipo funcional ocasionada por íleo paralitico nos bovinos. Assim, foi instituído um protocolo terapêutico com a finalidade de reestabelecer a função motora intestinal. Além da conduta terapêutica do pós operatório.

O A1 devido ao histórico de administração de medicamentos (VO) por parte do proprietário, aos achados de exame clínico e hematológico foi submetido a exame ultrassonográfico de região pulmonar. No exame, constatou-se irregularidades pleurais em ambos os campos pulmonares, diminuição da quantidade de reverberação e diversas imagens hiperecóicas, concentradas nas regiões crânio ventrais, sugestivas de pequenos abscessos, decorrentes possivelmente de uma broncopneumonia aspirativa.

O protocolo terapêutico e de suporte instituído nos animais durante o pós-operatório baseou-se em: A1 - Cloridrato de metoclopramida $(0,3 \mathrm{mg} / \mathrm{kg} / \mathrm{SC}$ - dose única), Cálcio $(250 \mathrm{ml} /$ IV - aplicação única), fluidoterapia intravenosa com solução fisiológica (5 litros/IV - aplicação única), fluido ruminal (10L/ VO) e suco de mandacaru (10L/VO), tulatromicina $(2,5 \mathrm{mg} / \mathrm{kg} /$ SC - duas doses intervaladas em 72 horas), Dipirona (25mg/ $\mathrm{kg} / \mathrm{IV}$ - duas doses intervaladas em 12 horas após a celiotomia e Meloxican (0,5mg/kg/IV - cinco doses SID); A2 - Cloridrato de metoclopramida (0,3mg/kg/SC - três doses SID), Cálcio $(200 \mathrm{ml} /$ IV - três aplicações SID), fluidoterapia intravenosa com solução fisiológica (7 litros/IV - aplicação única), fluido ruminal (10L/VO) e suco de mandacaru (10L/VO), oxitetraciclina $(20 \mathrm{mg} / \mathrm{kg} / \mathrm{IM}-$ três doses intervaladas em 72 horas), Flunixin niglumine $(2,2 \mathrm{mg} /$ $\mathrm{kg} / \mathrm{IV}$ - uma dose logo após a celiotomia) e Meloxican (0,5mg/ $\mathrm{kg} / \mathrm{IV}$ - quatro doses SID iniciado no dia posterior a celiotomia); A3 - Cloridrato de metoclopramida $(0,3 \mathrm{mg} / \mathrm{kg} / \mathrm{SC}$ - três doses SID), Cálcio (300 ml/IV - três aplicações SID), fluidoterapia intravenosa com solução fisiológica (5 litros/IV - seis dias), fluido ruminal (10L/VO), Gentamicina ( $4,0 \mathrm{mg} / \mathrm{kg} / \mathrm{IM}$ - oito aplicações, $24 / 24 \mathrm{~h})$, Flunixin niglumine $(2,2 \mathrm{mg} / \mathrm{kg} / \mathrm{IV}$ — cinco aplicações SID iniciado no dia da celiotomia).

Todos animais responderam positivamente ao tratamento instituído durante o pós-operatório, sendo restabelecidos os parâmetros fisiológicos, possuindo o animal A1 uma evolução clínica de 10 dias, enquanto para os A2 e A3 foram 12 dias. Após a alta médica os animais voltaram as suas respectivas propriedades, retornando à produção de leite, e não foi relatado recidiva do quadro clínico inicial ou aparecimento de outra enfermidade. A opção pela tulatromicina no A1, se decorreu devido ao quadro de pneumonia apresentado pelo mesmo, por ser um antibiótico macrólidio mais adequado para o caso clínico. Os demais animais seguiram o protocolo determinado pela CBG/UFRPE para pós operatório decorrente de celiotomia.

\section{Discussão}

Apesar de existirem relatos, que são poucos em bovinos e búfalos acometidos por íleo paralítico, a sua etiopatogenia não está bem definida. A condição em que há perda do tônus e motilidade como resultado da inibição do reflexo, segundo alguns autores, pode ocorrer nos casos de peritonite aguda, nas situações em que surge uma severa e prolongada distensão dos intestinos devido as obstruções intestinais e enterite, e quando existe excessiva manipulação das vísceras durante cirurgias abdominais, em que há dor (Pearson e Pinsent, 1977, Pradhan et al., 2008, Khalphallah et al., 2016; Desrochers e Anderson, 
2016, Constable et al., 2017, Fecteau et al., 2018). Em humanos, a disfunção do sistema nervoso autônomo parece ser primordial no desenrolar da dismotilidade intestinal pós-operatória, nesta condição, o sistema nervoso simpático, que geralmente é inibitório para o trato gastrointestinal, torna-se hiperativo. Em contrapartida, o efeito estimulante do nervo vago, que promove a liberação da acetilcolina no plexo mioentérico pode estar inibido (Goulart e Martins, 2010). Existe registro em vaca, com desfecho desfavorável, que esta desordem digestiva tenha ocorrido em função de uma ganglionite no mesentério, a qual comprometeu o plexo mioentérico (Auerbach) do intestino delgado e grosso, provocando com isso uma perda parcial do controle neural da peristalse (Baker et al., 1985). Há ainda relato da obstrução funcional com quadro de íleo paralítico, devido a uma severa parasitose por anfistomoses, acometendo o intestino delgado, em uma vaca, com resolução clínica favorável após o protocolo terapêutico (Yogeshpriya et al., 2011) Todavia, nenhuma destas condições foram constatadas nos casos descritos.

Quanto as vacas acometidas serem adultas e estarem no início da lactação, coincidem com relatos de Pearson e Pinsent (1977) e Francoz e Guard (2014), que relatam a ocorrência deste transtorno digestivo neste período de produção e recebendo dietas de elevada densidade energética, o que coincide com as dietas dos animais do presente estudo.

Segundo Francoz e Guard (2014), a condição clínica de íleo paralítico acometendo vacas leiteiras adultas apresentam uma sintomatologia variada, podendo chegar a mimetizar um quadro de obstrução intestinal completa. Em adição, Pearson e Pinsent (1977) e Constable et al. (2017) relataram que os principais sinais clínicos encontrados são anorexia, ausência de ruminação, hipomotilidade ruminal, presença de cólicas abdominais, redução ou ausência da eliminação das fezes, distensão da região abdominal direita com som de tilintar de líquidos ao balotamento, hipomotilidade ou atonia com distensão intestinal, variando de discreta a acentuada, impondo certa dificuldade no exame retal. Os animais do presente relato apresentaram sinais clínicos semelhantes, assim como segmentos intestinais dilatados durante a laparotomia exploratória, porém apenas no A3 foi observado cólicas abdominais. Destacou-se a pouca quantidade de fezes e a presença de muco na ampola retal. Neste contexto, Fecteau et al. (2018) comentam que poucas fezes podem ser encontradas na ampola retal nos primeiros dias após a obstrução intestinal mecânica ou funcional, sendo a presença de muco misturado às fezes um indicador de estase intestinal.

O resultado do hemograma apresentado pelo $\mathrm{A} 1$ está relacionado ao quadro pulmonar detectado no exame físico, possivelmente originado pela administração oral de medicamentos, de forma inadequada, praticada pelo produtor. Com relação ao animal A2 a resposta leucocitária pode estar associada a reação inflamatória insipiente encontrada, que pode ser atribuída a estase intestinal prolongada (Khalphallah et al., 2016). Quanto ao leucograma do A3 é sugestivo que animal tenha produzido uma resposta a um estímulo vacinal, devido à época coincidir com campanha vacinal na região. Em relação à análise de fluido ruminal, destacou-se o elevado teor de cloretos (> $30 \mathrm{mEq} / \mathrm{L}$ ) nos casos, evidenciando um refluxo de conteúdo abomasal rico em ácido clorídrico para o rúmen, devido ao comprometimento do trânsito aboral da ingesta, semelhante a outros tipos de distúrbios digestivos descritos em bovinos (Braun et al., 1990;
Afonso et al., 2008; Câmara et al., 2010; Silva et al., 2010; Silva et al., 2011; Silva et al., 2014).

Com relação às abordagens diagnósticas e cirúrgicas adotadas, segundo Pearson e Pinsent (1977) e Francoz e Guard (2014), a realização da laparotomia não é indicada imediatamente, já que a condição não é passível de alívio cirúrgico e pode ser tentado o tratamento conservativo. Porém, os autores também relatam que a manipulação das alças intestinais durante a laparotomia exploratória pode ocasionar um efeito benéfico na motilidade. Estudos conduzidos por Goulart e Martins (2010) na medicina humana se contrapõe e mostra um efeito inibitório na motilidade intestinal em casos de manipulação durante cirurgia, podendo o sucesso do tratamento dos animais citados está associado ao protocolo terapêutico empregado no pós operatório realizado nos animais.

A conduta terapêutica adotada no pós-operatório dos animais apresentou resultado satisfatório, a qual foi composta pela terapia antibiótica, analgésica e anti-inflamatória, correção da desidratação e do equilíbrio ácido-básico, reposição do cálcio, além da administração de fluido ruminal com a finalidade de recompor a microbiota ruminal. Ainda, enfatiza-se o do emprego do suco de mandacaru na qual atua como um agente emoliente sobre o conteúdo fecal e a utilização da metoclopramida como um agente prócinéticos. Nesta categoria, o emprego deste fármaco vem se destacando, por produzir um efeito satisfatório em ruminantes e por possuir um custo financeiro mais acessível quando comparado as outras opções de prócinéticos. (Roussel, 2004). No contexto clínico abordado, o uso deste prócinético, visando tratar quadros de hipotonia à atonia do trato gastrointestinal, vêm sendo utilizado com resultados favoráveis na clínica de ruminantes, auxiliando em restabelecer a motilidade ao padrão fisiológico (Spinosa et al., 2011).

Há de se comentar que Pradhan, Waghaye e Jadhave (2008) empregaram como conduta terapêutica, em um bovino acometido por este transtorno digestivo, a administração de sulfato de magnésio para ação laxativa (VO), bem como administração de soluções a base de dextrose e ringer lactato. Enquanto, Reddy e Sivajothi (2017) adotaram uma conduta parecida com a dos animais do presente estudo, utilizando antibioticoterapia associada com administração de cálcio e antinflamatorio. Em ambas situações houve sucesso.

\section{Conclusão}

Concluiu-se que o quadro de íleo paralítico, apesar de ter uma ocorrência baixa em bovinos, deve ser incluído como diagnóstico diferencial nos transtornos digestivos que comprometem total ou parcialmente o trânsito da ingesta. Ainda, ratifica-se a eficiência do protocolo terapêutico adotado, contendo cálcio, fluidoterapia , analgésicos e prócinéticos, que deverá ser usado inicialmente como base nestes tipos de caso. A utilização da ultrassonografia deve ser considerada uma ferramenta auxiliar como exame complementar para o diagnostico destes casos, visando identificação da presença ou ausência de motilidade intestinal e o diagnóstico diferencial com outras afecções que acometem o intestino. Quanto a etiologia da sua ocorrência não está bem definida os principais fatores de risco, entretanto há apenas o relato da sua ocorrência em vacas de leite em produção recebendo dietas com elevada densidade energética. Estudos de observação da sua ocorrência são necessários para suportar esta hipótese e outras que estejam associadas. 


\section{Referências}

AFONSO, J.A.B. Afecções intestinais em bovinos. Revista Acad. Ciênc. Anim, v.15, n.2, p.15 - 20, 2017.

AFONSO, J.A.B.; PEREIRA, A.L.L.; VIEIRA, A.C.S.; MENDONÇA, C.L.; COSTA, N.A.; SOUZA, M.I. Alterações clínicas e laboratoriais na obstrução gastrintestinal por fitobezoários em bovinos. Revista Brasileira de Saúde e Produção Animal, v. 9, n. 1, p. 91-102, 2008.

BRAUN, U.; STEINER, A.; KAEGI, B. Clinical, haematological and biochemical findings and the results of treatment in cattle with acute functional pyloric stenosis. Veterinary Record, v. 126, n. 3, p. 107-110, 1990.

BAKER, J.; HULLINGER R.L.; APPEL, G. Pospartum atony of the small and large intestine in a hosltein cow: A case of pseudoobstruction. Cornell Vet., v.75: p.289-296, 1985.

CAMARA, A.C.L.; AFONSO, J.A.B.; COSTA, N.A.; MENDONÇA, C.L.; SOUZA, M.I.; BORGES, J.R.J. Fatores de risco, achados clínicos, laboratoriais e avaliação terapêutica em 36 bovinos com deslocamento de abomaso. Pesquisa Veterinária Brasileira, v. 30, n. 5, p. 453-464, 2010.

CONSTABLE, P.D.; HINCHCLIFF, K.W.; DONE, S.H.; GRUNBERG, W. Veterinary Medicine: A textbook of the diseases of cattle, horses, sheep, pigs, and goats. 11 ed. United States of America: Saunders, 2017, 2278 p.

DESROCHERS, A.; ANDERSON, D.E. Intestinal Surgery. Vet Clin Food Anim, v. 32, n. 3, p. 645-671, 2016

DIRKSEN, G.; GRÜNDER, H.; STÖBER, M. Rosenberger: Exame Clínico dos Bovinos. 3. ed. Rio de Janeiro: Guanabara Koogan, 1993, $419 \mathrm{p}$.

FECTEAU, G.; DESROCHERS, A.; FRANCOZM, D.; NICHOLS, S. Diagnostic approach to the acute abdomen. Vet Clin Food Anim, v. 34, n. 1, p. 19-33, 2018.

FRANCOZ, D.; GUARD, C.L. Obstructive Intestinal Diseases. In: SMITH, B.P. (eds) Large Animal Internal Medicine. St Louis: Elsevier, 2014. P. 820-824.

FUBINI, SL.; DUCHARME, N.G. Farm Animal Surgery. 2nd Edition. St. Louis, Missouri: Saunders, 2017, 664p.

GOULART, D.; MARTINS, S. íleo paralítico pós-operatório: fisiopatologia, prevenção e tratamento. Rev Port Coloproct, v. 7, n. 2, p. 60-67, 2010.
HUSSAIN, S.; UPPAL, S.K.; RANDHAWA, C.S.; SOOD, N.K. Bovine intestinal obstruction:Blood gas analysis, serum C-reactive protein and clinical, haematological and biochemical alterations. J. Appl. Anim. Res., v. 43, n. 2, p. 224-230, 2015.

JAIN, N.C. Essentials of Veterinary Hematology. Philadelphia: Lead \& Febigar, 1993.

KHALPHALLAH, A; ELDIN, N.; ELMELIGY, E.; EL-HAWARI, S.F. Clinical and ultrasonographic observations of functional and mechanical intestinal ostruction in buffaloes (Bubalus bubalis). Veterinary Wordl, v.9, n.5, p. 475-480, 2016.

PEARSON, H.; PINSENT, P.J.N. Intestinal obstruction in cattle. Veterinary Record, v. 101, p. 162-166, 1977.

PRADHAN, M.S.; WAGHAYE, U.G.; JADHAVE, S.G. Paralytic ileus in a cattle. Veterinary World, v.1, n.6, p. 180, 2008.

REDDY, B.S.; SIVAJOTHI, S. Paralytic ileus due to Toxocara vitulorum in a calf and its management. Comparative Clinical Pathology, v. 26, n. 6, p. 1335-1338, 2017.

ROUSSEL, A.J. Postoperative Management. In: FUBINI, S.L.; DUCHARME, N.G. (Eds.) Farm Animal Surgery. St Louis: Saunders, 2004, p. 113-124.

SILVA, A.P.S.F.; AFONSO, J.A.B.; CORREIA, F.R.; DANTAS, A.F.; SOUZA, J.C.A.; DANTAS, A.C.; COSTA, N.A.; MENDONÇA, C.L. Obstrução intestinal por linfossarcoma em bovinos: Estudo retrospectivo. Veterinária e Zootecnia, v. 18, n. 2, p. 264-274, 2011.

SILVA, R.J; AFONSO, J.A.B.; COSTA, N.A.; MENDONÇA, C.L. Dilatação do ceco em bezerros: relato de casos. Revista Brasileira de Ciência Veterinária, v. 21, n. 2, p. 76-81, 2014.

SILVA, A.P.F.; AFONSO, J.A.B.; SOUZA, J.C.A.; COSTA, N.A.; MENDONÇA, C.L. Análise clínica e patológica em 20 casos de intussuscepção em bovinos. Veterinária e Zootecnia, v. 17, n. 3, p. 421-430, 2010.

SPINOSA, H.S.; GÓRNIAK, S.L.; BERNARDI, M.M. Farmacologia aplicada à medicina veterinária. Rio de Janeiro: Guanabara Koogan, 2011, 824p. YOGESHPRIYA, S.; AJITHKUMAR, S.; DHANESH, V.; KUTTY, R.R.; ALEX, P.C. Paralytic ileus due to amphistomosis in a cow. Journal of the Indian Veterinary Association, v.9, n.1, p. 48, 2011. 